\title{
On the Connectedness of Rational Arithmetic Discrete Hyperplanes
}

\author{
Damien Jamet ${ }^{1}$ and Jean-Luc Toutant ${ }^{2}$ \\ ${ }^{1}$ LORIA - INRIA Lorraine \\ 615 rue du Jardin Botanique - BP 101 \\ F-54602 Villers-lès-Nancy, France \\ jamet@loria.fr \\ ${ }^{2}$ LIRMM - CNRS UMR 5506 - Université Montpellier II \\ 161 rue Ada - 34392 Montpellier Cedex 5, France \\ toutant@lirmm.fr
}

\begin{abstract}
While connected arithmetic discrete lines are entirely characterized by their arithmetic thickness, only partial results exist for arithmetic discrete hyperplanes in any dimension. In the present paper, we focus on 0 -connected rational arithmetic discrete planes in $\mathbb{Z}^{3}$. Thanks to an arithmetic reduction on a given integer vector $\mathbf{n}$, we provide an algorithm which computes the thickness of the thinnest 0-connected arithmetic plane with normal vector $\mathbf{n}$.
\end{abstract}

\section{Introduction}

In [1], J.-P. Reveillès initiated a new approach of linear discrete objets and introduced arithmetic discrete lines as sets of pairs of integers satisfying a double Diophantine inequality : the arithmetic discrete line with normal vector $\mathbf{n} \in \mathbb{R}^{2}$, translation parameter $\mu \in \mathbb{R}$ and thickness $w \in \mathbb{R}$ is the set $\mathbf{D}(\mathbf{n}, \mu, w)=$ $\left\{\mathbf{x} \in \mathbb{Z}^{2}, 0 \leq \mathbf{n} \cdot \mathbf{x}+\mu<w\right\}$, where $\mathbf{n} \cdot \mathbf{x}=n_{1} x_{1}+n_{2} x_{2}$ is the usual Euclidean scalar product of $\mathbf{n}$ and $\mathbf{x}$. Geometrically, an arithmetic discrete line can be viewed as a set of integer points of the plane $\mathbb{R}^{2}$ included in a band delimited by two parallel Euclidean lines (see Fig. 1). The thickness parameter $w$ plays a key role in the topology of the arithmetic discrete lines: given $\mathbf{n} \in \mathbb{R}^{2}$ and $\mu \in \mathbb{R}$, the thinnest 0 -connected (resp. 1-connected) arithmetic discrete line among the ones with normal vector $\mathbf{n}$ and translation parameter $\mu$ is the arithmetic discrete line $\mathbf{D}\left(\mathbf{n}, \mu,\|\mathbf{n}\|_{\infty}\right)$ (resp. $\mathbf{D}\left(\mathbf{n}, \mu,\|\mathbf{n}\|_{1}\right)$ ) (see Section 2 for the definition of the 0-connectedness and 1-connectedness) [1].

The definition of arithmetic discrete lines extends naturally in dimension 3 to the arithmetic discrete planes and in any dimension $d \geq 2$ to the arithmetic discrete hyperplanes [2. It is thus natural to try to exhibit a similar relation between the $\kappa$-connectedness of an arithmetic discrete hyperplane and its thickness. In fact, the 2-dimensional case is somewhat confusing since a 0-connected (resp. 1connected) arithmetic discrete line is also 1-separating (resp. 1-separating) in $\mathbb{Z}^{2}$ (see Section 2). 

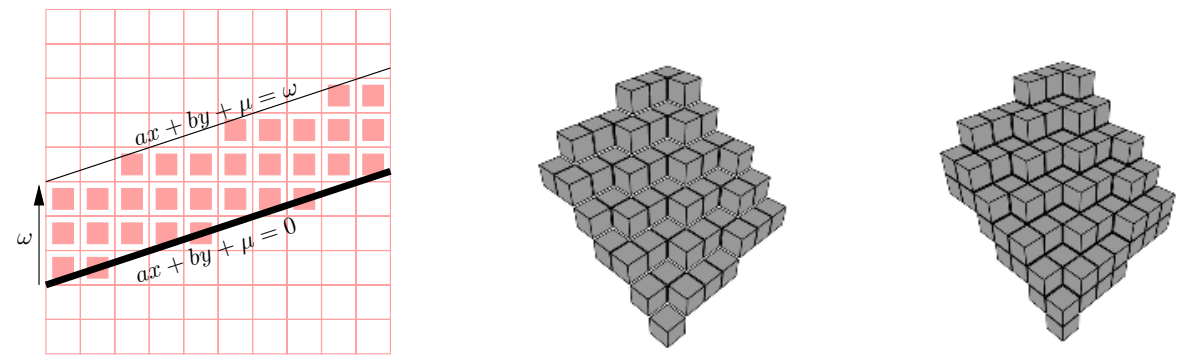

Fig. 1. From left to right: an arithmetic discrete line - a naive discrete plane - a standard discrete plane

In the particular case of rational arithmetic discrete hyperplanes (rememeber that an arithmetic discrete hyperplane is rational if its normal vector $\mathbf{n} \in \mathbb{R}^{d}$ is colinear to an integer vector, or equivalently, if the $\mathbb{Q}$-vector space spanned by $\left\{n_{1}, \ldots, n_{d}\right\}$ is of dimension 1), several approaches have been attempted 223]4 although none of them provides an explicit formula to compute the thickness of the thinnest 0 -connected rational arithmetic discrete hyperplane with any given normal vector.

In 4, V. Brimkov and R. Barneva partially solved this request for rational arithmetic discrete planes whose the normal vector $\mathbf{n} \in \mathbb{Z}^{2}$ satisfies particular conditions (for instance when $\left|n_{1}\right|+2\left|n_{2}\right| \leq\left|n_{3}\right|$ ) and provided an algorithm for the entire problem. Unfortunately, their algorithm seems to incorrect and does not generally return the right thickness (see Section 4).

In 3, Y. Gérard investigated a problem close to the one we are interested in in the present paper: given an arithmetic discrete hyperplane $\mathbf{P}(\mathbf{n}, \mu, w)$ and $\kappa \in\{0, \ldots, d-1\}$, is $\mathbf{P}(\mathbf{n}, \mu, w) \kappa$-connected ? In other words, given the graph $\mathbf{G}(\mathbf{n}, \mu, w)$ whose vertices are the points of $\mathbf{P}(\mathbf{n}, \mu, w)$ and whose edges are the pairs $\{\mathbf{x}, \mathbf{y}\}$ of $\kappa$-adjacent points of $\mathbf{P}(\mathbf{n}, \mu, w)$, does $\mathbf{G}(\mathbf{n}, \mu, w)$ admit a unique connected component? The main difficulty of this problem is the possibly infiniteness of $\mathbf{G}(\mathbf{n}, \mu, w)$. Assuming $\operatorname{dim}_{\mathbb{Q}}\left\{n_{1}, \ldots, n_{d}\right\}=1$, one reduces $\mathbf{G}(\mathbf{n}, \mu, w)$ to a finite graph by quotienting $\mathbf{G}(\mathbf{n}, \mu, w)$ iteratively by a subgroup of rank 1 of the lattice of periods of $\mathbf{P}(\mathbf{n}, \mu, w)$. Since $\mathbf{G}(\mathbf{n}, \mu, w)$ is injectively projectable in $\mathbb{Z}^{d}$, then, with at most $d$ such quotienting processes, one reduces $\mathbf{G}(\mathbf{n}, \mu, w)$ to a finite graph with the same connectedness as $\mathbf{G}(\mathbf{n}, \mu, w)$.

In the present paper, we deal with the determination of the thickness of the thinnest 0-connected rational arithmetic discrete plane with a given normal vector $\mathbf{n}$. For this purpose, we give a short and elementary algorithm which takes a vector $\mathbf{n} \in \mathbb{Z}^{3}$ as entry and returns the thickness $w$ of the thinnest 0-connected arithmetic discrete plane with normal vector $\mathbf{n}$. While Y. Gérard, V. Brimkov and R. Barneva's approaches need to determine a connected component, our algorithm is entirely arithmetic and does not need to consider any connectivity graph.

Here is the sketch of the present paper. Section 2 is devoted to the basic notions useful for the remaining. In Section 3, we investigate the notions 
of $\kappa$-connectedness and $\kappa$-separatingness and state a first comparison between their characterization in the case of rational arithmetic discrete lines and rational arithmetic discrete hyperplanes. In Section 4, we focus on V. Brimkov and R. Barneva's investigation [4]. After having recalled some of their results, we exhibit a counter example of the algorithm they proposed. In Section 5 , we introduce an arithmetic reduction on the integer vectors preserving the 0 connectedness of arithmetic discrete planes. We end this section by designing an elementary and quite short algorithm which computes the minimal thickness by iterating this arithmetic reduction.

\section{Basic Notions}

The aim of this section is to introduce the basic notions and definitions we use throughout the present paper.

Let $d$ be an integer equal or greater than 2 and let $\left\{\mathbf{e}_{\mathbf{1}}, \ldots, \mathbf{e}_{\mathbf{d}}\right\}$ denote the canonical basis of the Euclidean vector space $\mathbb{R}^{d}$. Let us call a discrete set any subset of the discrete space $\mathbb{Z}^{d}$. In the following, for the sake of clarity, we denote by $\left(x_{1}, \ldots, x_{d}\right)$ the point (resp. vector) $\mathbf{x}=\sum_{i=1}^{d} x_{i} \mathbf{e}_{\mathbf{i}} \in \mathbb{R}^{d}$. An integer point $\mathbf{x} \in \mathbb{Z}^{d}$ is called a voxel (resp. a pixel if $d=2$ ). A subset of $\mathbb{Z}^{d}$ is called a discrete set.

Let $\kappa \in\{0, \ldots, d-1\}$. Two voxels $\mathbf{x} \in \mathbb{Z}^{d}$ and $\mathbf{x}^{\prime} \in \mathbb{Z}^{d}$ are said to be $\kappa$ adjacent if $\left\|\mathbf{x}-\mathbf{x}^{\prime}\right\|_{\infty}=1$ and $\left\|\mathbf{x}-\mathbf{x}^{\prime}\right\|_{1} \leq d-\kappa$. In other words, $\mathbf{x} \in \mathbb{Z}^{d}$ and $\mathbf{x}^{\prime} \in \mathbb{Z}^{d}$ are $\kappa$-adjacent if they are distinct, the differences of their coordinates are at most 1 and $\mathbf{x}$ and $\mathbf{x}^{\prime}$ have at most $d-\kappa$ different coordinates (resp. at least $\kappa$ identical components). A $\kappa$-path is a (finite or infinite) sequence of consecutive $\kappa$-adjacent voxels. If $\left(\gamma_{i}\right)_{1 \leq i \leq n}$ is a finite $\kappa$-path, then we say that $\gamma$ links the voxel $\gamma_{1}$ to the voxel $\gamma_{n}$. A subset $E \subseteq \mathbb{Z}^{d}$ is said $\kappa$-connected if, for each pair of voxels $\left(\mathbf{x}, \mathbf{x}^{\prime}\right) \in E^{2}$, there exists a $\kappa$-path in $E$ linking $\mathbf{x}$ to $\mathbf{x}^{\prime}$. Given a discrete set $E \subseteq \mathbb{Z}^{d}$ and given $\kappa \in\{0, \ldots, d-1\}$, one says that $E$ is $\kappa$-separating in $\mathbb{Z}^{d}$ if its complement in $\mathbb{Z}^{d}$ has (at least) two $\kappa$-connected components.

In [1], J.-P. Reveillès introduced the arithmetic discrete line as a set of integer points satisfying a double Diophantine inequality. This definition extends in a natural way to higher dimensions:

Definition 1 (Arithmetic discrete hyperplane [1,2]). The arithmetic discrete hyperplane with normal vector $\mathbf{n} \in \mathbb{Z}^{d}$, translation parameter $\mu \in \mathbb{Z}$ and thickness $w \in \mathbb{Z}$ is the discrete set $\mathbf{P}(\mathbf{n}, \mu, w)$ defined by:

$$
\mathbf{P}(\mathbf{n}, \mu, w)=\left\{\mathbf{x} \in \mathbb{Z}^{d}, 0 \leq \mathbf{n} \cdot \mathbf{x}+\mu<w\right\}
$$

where $\mathbf{n} \cdot \mathbf{x}$ denotes the usual Euclidean scalar product in $\mathbb{R}^{d}$. If $w=\|\mathbf{n}\|_{\infty}$ (resp. $w=\|\mathbf{n}\|_{1}$ ) then $\mathbf{P}(\mathbf{n}, \mu, w)$ is said naive (resp. standard). If $d=2$ the arithmetic discrete hyperplane $\mathbf{P}(\mathbf{n}, \mu, w)$ is called an arithmetic discrete line and is denoted by $\mathbf{D}(\mathbf{n}, \mu, w)$. If $d=3$ the arithmetic discrete hyperplane $\mathbf{P}(\mathbf{n}, \mu, w)$ is called an arithmetic discrete plane. 
Remark 1. Throughout the present paper, when $\mathbf{P}(\mathbf{n}, \mu, w)$ is a rational arithmetic hyperplane, we assume, with no loss of generality, that $\operatorname{gcd}\left\{n_{1}, \ldots, n_{d}\right\}=$ $1, \mu \in \mathbb{Z}$ and $w \in \mathbb{Z}$. Moreover, since the isometry group of the unit cube $[-0.5,0.5]^{d}$ acts on the set of arithmetic discrete hyperplanes and since any isometry of $[-0.5,0.5]^{d}$ preserves the $\kappa$-connectedness of any arithmetic discrete hyperplane, whatever $\kappa \in\{0, \ldots, d-1\}$, then in the following, except when explicitly mentioned, we suppose the normal vector $\mathbf{n} \in \mathbb{Z}^{d}$ to satisfy $0 \leq n_{1} \leq \cdots \leq n_{d}$.

In Section 3, we recall some partial results on the connectedness of arithmetic discrete lines and give a first extension of them to arithmetic discrete hyperplanes.

\section{$3 \kappa$-Connected Arithmetic Discrete Lines vs. $\kappa$-Separating Arithmetic Discrete Hyperplanes}

Let us first deal with the case $d=2$. In [1], J.-P. Reveillès showed how the $\kappa$-connectedness of an arithmetic discrete line depends only on its normal vector and its thickness:

Theorem 1 ([1]). Let $\mathbf{D}(\mathbf{n}, \mu, w)$ be the arithmetic discrete line with normal vector $\mathbf{n} \in \mathbb{Z}^{2}$, translation parameter $\mu \in \mathbb{Z}$ and thickness $w \in \mathbb{Z}$. Then $\mathbf{D}(\mathbf{n}, \mu, w)$ is 0-connected (resp. 1-connected) if and only if $w \geq\|\mathbf{n}\|_{\infty}$ (resp. $\left.w \geq\|\mathbf{n}\|_{1}\right)$.

It becomes natural to try to extend Theorem 1 to higher dimensions, that is, given $\mathbf{n} \in \mathbb{Z}^{d}, \mu \in \mathbb{Z}$ and $\kappa \in\{0, \ldots, d-1\}$, to try to characterize the thickness of the thinnest $\kappa$-connected arithmetic discrete hyperplane with normal vector $\mathbf{n}$ and translation parameter $\mu$.

Let us give a helpful reduction of our problem: if $\mu \in \mathbb{Z}$ and $\mathbf{n} \in \mathbb{Z}^{d}$, then the $\kappa$-connectedness (resp. $\kappa$-separatingness in $\mathbb{Z}^{d}$ ) of $\mathbf{P}(\mathbf{n}, \mu, w)$, whatever $d \geq 2$ and $\kappa \in\{0, \ldots, d-1\}$, does not depend on the translation parameter $\mu$. Indeed, it is a direct consequence of the following lemma:

Lemma 1. Let $\mathbf{P}(\mathbf{n}, \mu, w)$ be an arithmetic discrete hyperplane with $d \geq 2$, $\mu \in \mathbb{Z}$ and $\mathbf{n} \in \mathbb{Z}^{d}$. For all $\mu^{\prime} \in \mathbb{Z}$, there exists a vector $\boldsymbol{\alpha} \in \mathbb{Z}^{d}$ such that $\mathbf{P}(\mathbf{n}, \mu, w)=\mathbf{P}\left(\mathbf{n}, \mu^{\prime}, w\right)+\boldsymbol{\alpha}$.

Proof. It obviously follows form Bezout's Lemma applied on the coordinates of $\mathbf{n}$.

From now on, we consider only rational arithmetic discrete hyperplanes with a null translation parameter. Thanks to Lemma 1, in the determination of the thickness of the thinnest arithmetical discrete hyperplane with a given rational normal vector, this assumption is not restrictive. From now on, in order to simplify the notation, we denote by $\mathbf{P}(\mathbf{n}, w)$ the arithmetic discrete hyperplane with normal vector $\mathbf{n}$, translation parameter 0 and thickness $w$. 
Definition 2 ( $\kappa$-Connecting thickness). Let $\mathbf{n} \in \mathbb{Z}^{d}$ and $\kappa \in\{0, \ldots, d-1\}$. The thickness $w_{\kappa}$ of the thinnest $\kappa$-connected arithmetic discrete hyperplane with normal vector $\mathbf{n}$ is called the $\kappa$-connecting thickness of $\mathbf{n}$.

Let us now investigate the $\kappa$-connectedness of arithmetic discrete planes $(d=3)$. It is not difficult to exhibit a 0 -connected arithmetic discrete plane $\mathbf{P}(\mathbf{n}, w)$ thinner than the naive one, that is, satisfying $w<\|\mathbf{n}\|_{\infty}$ (see Fig. 2). Similarly, one easily finds a 2-connected arithmetic discrete plane $\mathbf{P}(\mathbf{n}, w)$ thinner than the standard one, that is, with $w<\|\mathbf{n}\|_{1}$.

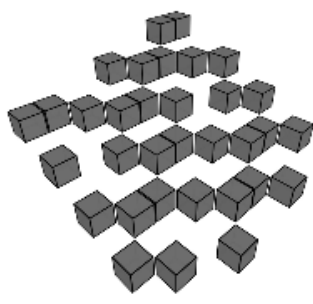

(a) A 0-connected arithmetic discrete plane thinner than the naive one

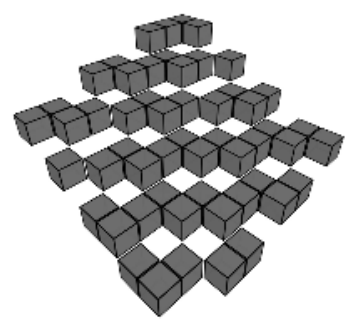

(b) A 1-connected arithmetic discrete plane thinner than the standard one

Fig. 2. Connected arithmetic discrete planes

Nevertheless, although Theorem 1 does not seem to extend naturally to higher dimensions, it admits a quite nice generalization of it concerning the $\kappa$-separating arithmetic discrete hyperplane. For the sake of clarity, we introduce the following notation, providing a norm on $\mathbb{R}^{d}$ :

Notation. - Let $\mathbf{x} \in \mathbb{R}^{d}$ and let $\sigma$ be a permutation over the set $\{1, \ldots, d\}$ such that, for all $i \in\{1, \ldots, d-1\},\left|x_{\sigma(i)}\right| \leq\left|x_{\sigma(i+1)}\right|$. For all $\kappa \in\{0, \ldots, d-1\}$, we denote by $] \mathbf{x}\left[_{\kappa}\right.$ the following number:

$$
] \mathbf{x}\left[_{\kappa}=\sum_{i=d-\kappa}^{d}\left|x_{\sigma(i)}\right| .\right.
$$

In other words, $] \mathbf{x}[\kappa$ is equal to the sum of the $(\kappa+1)$ greatest absolute values of the coordinates of $\mathbf{x}$.

One checks that, for each $\kappa \in\{0, \ldots, d-1\}$, the map $] \cdot{ }_{\kappa}: \mathbb{R}^{d} \longrightarrow \mathbb{R}^{d}$ is a norm on $\mathbb{R}^{d}$. Moreover, one has $] \cdot\left[{ }_{0}=\|\cdot\|_{\infty}\right.$ and $] \cdot\left[{ }_{d-1}=\|\cdot\|_{1}\right.$.

In the particular case of $d=2$, for $\kappa \in\{0,1\}$, the $\kappa$-connected arithmetic discrete lines are exactly the $(2-(\kappa+1))$-separating ones in $\mathbb{Z}^{2}$ and Theorem 1 is reformulated as follows:

Theorem 2 ([1]). Let $\mathbf{D}(\mathbf{n}, w)$ be the arithmetic discrete line with normal vector $\mathbf{n} \in \mathbb{Z}^{2}$ and thickness $w \in \mathbb{Z}$. Let $\kappa \in\{0,1\}$. Then, $\mathbf{D}(\mathbf{n}, w)$ is $(1-\kappa)$ separating in $\mathbb{Z}^{2}$ if and only if $\left.w \geq\right] \mathbf{n}[\kappa$. 
In fact, as previously mentioned, the $\kappa$-separatingness of an arithmetic discrete hyperplane $\mathbf{P}(\mathbf{n}, w)$, whatever the dimension $d$, is entirely characterized by $] \mathbf{n}\left[{ }_{\kappa}\right.$. Indeed, Theorem 2 extends in the most natural way to every dimension:

Theorem 3 ([2]). Let $\mathbf{P}(\mathbf{n}, w)$ be the arithmetic discrete hyperplane with normal vector $\mathbf{n} \in \mathbb{Z}^{d}$ and thickness $w \in \mathbb{Z}$. Let $\kappa \in\{0, \ldots, d-1\}$. The arithmetic discrete hyperplane $\mathbf{P}(\mathbf{n}, w)$ is $(d-\kappa-1)$-separating in $\mathbb{Z}^{d}$ if and only if $\left.w \geq\right] \mathbf{n}[\kappa$.

\section{V. Brimkov and R. Barneva's Investigation: An Algorithmic Approach [4]}

In [4, V. Brimkov and R. Barneva investigated 0-connected rational arithmetic discrete planes. They explicitly provided the 0-connecting thickness of some vectors $\mathbf{n} \in \mathbb{Z}^{3}$ and an algorithm for computing it in the general case. In the present section, we exhibit a counter-example to this algorithm and deduce that it does not always return the correct output.

Let $\mathbf{P}(\mathbf{n}, w)$ be a rational arithmetic discrete plane. It is well known that if $w \geq\|\mathbf{n}\|_{\infty}$ then $\mathbf{P}(\mathbf{n}, w)$ is 0 -connected (see 2] Cor. 10 p. 307). Hence, if $w_{0}$ is the 0 -connecting thickness of $\mathbf{n}$, then $w_{0} \leq\|\mathbf{n}\|_{\infty}$. In [4], V. Brimkov and R. Barneva reduced the determination of $w_{0}$ to the determination of the 0-connectedness of a subset of $\mathbb{Z}^{2}$ as follows:

Theorem 4 ([4]). Let $\mathbf{P}(\mathbf{n}, w)$ be a rational arithmetic discrete plane with $\|\mathbf{n}\|_{\infty}=\left|v_{3}\right|$ and $w \leq\|\mathbf{n}\|_{\infty}$. The arithmetic dscrete plane $\mathbf{P}(\mathbf{n}, w)$ is 0 -connected in $\mathbb{Z}^{3}$ if and only if the set $\left\{\mathbf{x} \in \mathbb{Z}^{2}, v_{1} x_{1}+v_{2} x_{2} \bmod v_{3} \in[0, w[\}\right.$ is 0 -connected in $\mathbb{Z}^{2}$.

Remark 2. Let us remember that, thanks to Remark 1, the condition $\|\mathbf{n}\|_{\infty}=$ $\left|v_{3}\right|$ in Theorem 4 is not restrictive. Up to an isometry, one can similarly treat the cases $\|\mathbf{n}\|_{\infty}=\left|v_{1}\right|$ and $\|\mathbf{n}\|_{\infty}=\left|v_{2}\right|$.

For the sake of clarity, we introduce the following notation:

Notation. - Let $\mathbf{P}(\mathbf{n}, w)$ be an arithmetic discrete plane with $\|\mathbf{n}\|_{\infty}=\left|v_{3}\right|$ and $w \leq\|\mathbf{n}\|_{\infty}$. We denote by $\boldsymbol{\Pi}(\mathbf{n}, w)$ the set $\left\{\mathbf{x} \in \mathbb{Z}^{2}, v_{1} x_{1}+v_{2} x_{2} \bmod v_{3} \in[0, w[\}\right.$. In what follows, since $\boldsymbol{\Pi}(\mathbf{n}, w)$ can be indexed by (a subset of) $\mathbb{Z}^{2}$, we call $\mathbf{\Pi}(\mathbf{n}, w)$ the array of remainders of $\mathbf{P}(\mathbf{n}, w)$. For $\mathbf{x} \in \mathbb{Z}^{2}$, the number $v_{1} x_{1}+v_{2} x_{2} \bmod v_{3}$ is called the remainder of $\mathbf{x}$. Let us notice that this denomination is not exactly the one used in [45], but is equivalent in the way we use it.

With this notation, from Theorem 4, it follows:

Corollary 1. Let $\mathbf{P}(\mathbf{n}, w)$ be a rational arithmetic discrete plane with $\|\mathbf{n}\|_{\infty}=$ $\left|v_{3}\right|$ and $w \leq\|\mathbf{n}\|_{\infty}$. The arithmetic dscrete plane $\mathbf{P}(\mathbf{n}, w)$ is 0 -connected in $\mathbb{Z}^{3}$ if and only if the set $\mathbb{Z}^{2} \backslash \mathbf{\Pi}(\mathbf{n}, w)$ is not 0 -separating in $\mathbb{Z}^{2}$. 
Before describing V. Brimkov and R. Barneva's algorithm, let us introduce a notation:

Notation. - Let $\mathbf{n} \in \mathbb{Z}^{3}$ such that $0 \leq n_{1} \leq n_{2} \leq n_{3}$ and $\operatorname{gcd}\left\{n_{1}, n_{2}, n_{3}\right\}=$ 1. We denote by $\boldsymbol{\Gamma}(\mathbf{n})$ the set of 1 -paths in $\boldsymbol{\Pi}\left(\mathbf{n},\|\mathbf{n}\|_{\infty}\right)$ linking two points of maximal remainder, that is, $n_{3}-1$. For a 1 -path $\gamma \in \boldsymbol{\Gamma}(\mathbf{n})$, we denote by

$$
\min (\gamma)=\min \left\{n_{1} i_{1}+n_{2} i_{2} \quad \bmod n_{3},\left(i_{1}, i_{2}\right) \in \gamma\right\}
$$

In other words, $\min (\gamma)$ is the smallest remainder reached in $\gamma$.

In [4, V. Brimkov and R. Barneva stated:

Theorem 5 ([4]). Let $\mathbf{n} \in \mathbb{Z}^{3}$ such that $0 \leq n_{1} \leq n_{2} \leq n_{3}$ and $\operatorname{gcd}\left\{n_{1}, n_{2}, n_{3}\right\}=1$. Let $w_{0} \in \mathbb{Z}$ be the 0 -connecting thickness of $\mathbf{n}$. Then $w_{0}=\max \{\min (\gamma) \in \Gamma(\mathbf{n})\}+1$.

Given a vector $\mathbf{n} \in \mathbb{Z}^{3}$ satisfying $0 \leq n_{1} \leq n_{2} \leq n_{3}$ and $\operatorname{gcd}\left\{n_{1}, n_{2}, n_{3}\right\}=$ 1 , the problem of determining $w_{0}$ can thus be reduced to the following one: how to compute $\max \{\min (\gamma) \in \Gamma(\mathbf{n})\}$ in a reasonable time ? V. Brimkov and R. Barneva assumed that only exclusively down-right or up-right searches (with additional conditions) in $\Pi\left(\mathbf{n},\|\mathbf{n}\|_{\infty}\right)$ are necessary to compute $w_{0}$ (see [4]). This assertion is false and here is a counter-example:

Example 1. Let $\mathbf{n}=(4,7,16)$. Let $w_{0}$ be the 0 -connecting thickness of $\mathbf{n}$. In Figure 3(a), both light paths are computed by V. Brimkov and R. Barneva's algorithm. Minimal remainders of each one are respectively 3 and 5 , and the algorithm returns $w_{0}=\max \{3,5\}+1=6$. In Figure 3(b), one sees that $\Pi(\mathbf{n}, 6)$ is not 0-connected, and by Theorem 4. so is $\mathbf{P}(\mathbf{n}, 6)$. In fact, the correct 0connecting thickness for the vector $\mathbf{n}$ is 7 as shown in Figure [3. (c). This value is obtained with the dark grey path in Figure 3(a), which cannot be computed using exclusively up-right or down-right searches.

\section{Arithmetic Reduction of an Arithmetic Discrete Plane}

We have seen in Section 4 that V. Brimkov and R. Barneva's algorithm needs a graph traversal for computing the 0-connecting thickness of a given integer vector. Similarly, Y. Gérard proposed an algorithm, based on a graph traversal too, testing whether a given rational arithmetic discrete hyperplane is $\kappa$-connected. In the present section, we propose a reduction acting on the normal vector and the arithmetic thickness of an arithmetic discrete plane $\mathbf{P}(\mathbf{n}, w)$ which returns an arithmetic discrete plane $\mathbf{P}\left(\mathbf{n}^{\prime}, w^{\prime}\right)$ with the same 0-connectedness as $\mathbf{P}(\mathbf{n}, w)$ and such that $\left|n_{1}^{\prime}\right|<\left|n_{1}\right|$. By iterating this reduction, we obtain in a finite time an arithmetic discrete plane $\mathbf{P}\left(\mathbf{n}^{\prime}, w^{\prime}\right)$ with a zero coordinate. The 0 -connecting thickness (see Definition 2) of such a vector is easy to determine:

Lemma 2. Let $\mathbf{P}(\mathbf{n}, w)$ be a rational arithmetic discrete plane. Let us suppose there exists $i \in\{1,2,3\}$ such that $n_{i}=0$. Then, $\mathbf{P}(\mathbf{n}, w)$ is 0 -connected if and only if $w \geq\|\mathbf{n}\|_{\infty}$. In other words, the 0 -connecting thickness of $\mathbf{n}$ is $\|\mathbf{n}\|_{\infty}$. 


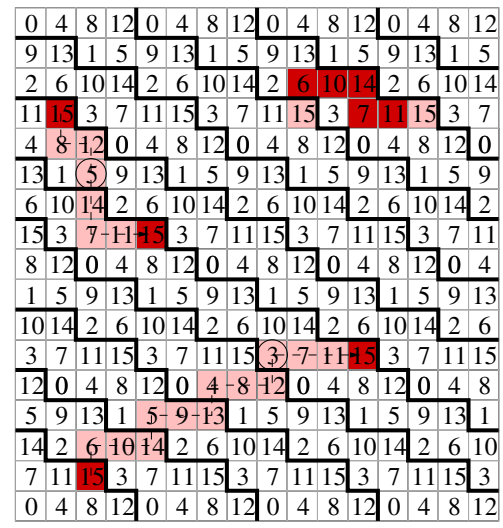

(a) 1-connected paths in the 2-dimensional representation

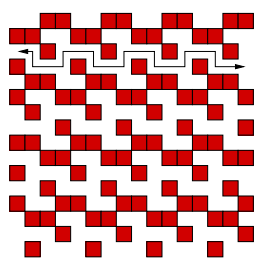

(b) Array of remainders $\boldsymbol{\Pi}(\mathbf{n}, 6)$

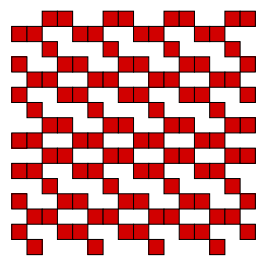

(c) Array of remainders $\Pi(\mathbf{n}, 7)$

Fig. 3. Computation of V. Brimkov and R. Barneva's algorithm [4] on the vector $\mathbf{n}=(4,7,16)$

Proof. It is well known that, if $w \geq\|\mathbf{n}\|_{\infty}$ then $\mathbf{P}(\mathbf{n}, w)$ is 0-connected [2]. Conversely, let us suppose, with no loss of generality, that $n_{1}=0$ and $0 \leq n_{2} \leq$ $n_{3}$. Let $\mathbf{x} \in \mathbb{Z}^{2}$ such that $n_{1} x_{1}+n_{2} x_{2}=n_{2} x_{2} \equiv n_{3}-1 \bmod n_{3}$ (remember we assume $\left.\operatorname{gcd}\left\{n_{1}, n_{2}, n_{3}\right\}=1\right)$. Then, for all $k \in \mathbb{Z},\left(x_{1}+k\right) n_{1}+x_{2} n_{2}=$ $x_{1} n_{1}+x_{2} n_{2} \equiv n_{3}-1 \bmod n_{3}$. Hence, for all $k \in \mathbb{Z},\left(x_{1}+k, x_{2}\right) \in \mathbf{\Pi}(\mathbf{n}, w)$ and $\boldsymbol{\Pi}(\mathbf{n}, w)$ is not 0 -connected. The result follows from Theorem 4 .

Remember that, thanks to Theorem 4, one can reduce the determination of the 0-connectedness of the arithmetic discrete plane $\mathbf{P}(\mathbf{n}, w)$ to the one of $\mathbf{\Pi}(\mathbf{n}, w)=$ $\left\{\mathbf{x} \in \mathbb{Z}^{2}, n_{1} x_{1}+n_{2} x_{2} \bmod n_{3} \in\left[0, w[\}\right.\right.$ with $\mathbf{n} \in \mathbb{Z}^{3}$ and $n_{3}=\|\mathbf{n}\|_{\infty}$. Moreover, a direct consequence of Theorem 4 is:

Lemma 3 (Symmetry Lemma [4]). Let $\Omega: \mathbb{N}^{3} \rightarrow \mathbb{N}$ be the function mapping each vector of $\mathbb{N}^{3}$ to its 0 -connecting thickness. For all $\mathbf{n} \in \mathbb{Z}^{3}$, if $0 \leq n_{1}, n_{2} \leq n_{3}$, then $\Omega\left(n_{1}, n_{2}, n_{3}\right)=\Omega\left(n_{3}-n_{1}, n_{2}, n_{3}\right)=\Omega\left(n_{1}, n_{3}-n_{2}, n_{3}\right)=\Omega\left(n_{3}-n_{1}, n_{3}-\right.$ $\left.n_{2}, n_{3}\right)$.

Given a vector $\mathbf{n} \in \mathbb{Z}^{3}$, thanks to Lemma 3 and to the action of the isometry group of the cube on the set of arithmetic discrete planes, one suppose with no loss of generality and in order to compute the 0 -connecting thickness of $\mathbf{n}$ that $0 \leq 2 n_{1} \leq 2 n_{2} \leq n_{3}$.

Let us now state the main theorem of the present section:

Theorem 6 (Arithmetic reduction). Let $\mathbf{n} \in \mathbb{Z}^{3}$ such that $0 \leq 2 n_{1} \leq 2 n_{2} \leq$ $n_{3}$ and let $w \in \mathbb{Z}$. Let $(q, r) \in \mathbb{N}^{2}$ be the unique pair of integers such that $n_{2}=q n_{1}+r$ and $r \in\left[0, n_{1}\left[\right.\right.$. Let $\mathbf{n}^{\prime}=M \cdot \mathbf{n}$ with 


$$
M=\left(\begin{array}{rrr}
1 & 0 & 0 \\
-q & 1 & 0 \\
1-q & -1 & 1
\end{array}\right)
$$

and let $w^{\prime}=w-\left(n_{2}-n_{1}\right)$. Then, the arithmetic discrete plane $\mathbf{P}(\mathbf{n}, w)$ is 0 -connected if and only if so is the arithmetic discrete plane $\mathbf{P}\left(\mathbf{n}^{\prime}, w^{\prime}\right)$.

In order to prove Theorem 6, let us introduce in some sense the dual notion of the $\kappa$-connecting thickness of a vector:

Definition 3 ( $\kappa$-separating thickness). Let $\mathbf{n} \in \mathbb{Z}^{3}$ and let $\kappa \in\{0,1\}$. The $\kappa$-separating thickness $\bar{w}_{\kappa}$ of $\mathbf{n}$ is the thickness of the thinnest $\kappa$-separating $\Pi(\mathbf{n}, w)$, with $w \in \mathbb{Z}$.

An easy computation directly gives:

Lemma 4. Let $\mathbf{n} \in \mathbb{Z}^{3}$ such that $0 \leq n_{1}, n_{2} \leq n_{3}$ and $\operatorname{gcd}\left\{n_{1}, n_{2}, n_{3}\right\}=1$. Let $w_{0}$ (resp. $\bar{w}_{0}$ ) be the 0-connecting thickness (resp. 0-separating thickness) of $\mathbf{n}$. Then $w_{0}+\bar{w}_{0}=n_{3}+1$.

Proof. Let $w \in \mathbb{N}$. Then

$$
\begin{aligned}
\mathbb{Z} \backslash \boldsymbol{\Pi}(\mathbf{n}, w) & =\left\{\left(x_{1}, x_{2}\right) \in \mathbb{Z}^{2}, n_{1} x_{1}+n_{2} x_{2} \bmod n_{3} \in\left[w, n_{3}[\}\right.\right. \\
& =\left\{\left(x_{1}, x_{2}\right) \in \mathbb{Z}^{2}, n_{1} x_{1}+n_{2} x_{2}-w \bmod n_{3} \in\left[0, n_{3}-w[\}\right.\right.
\end{aligned}
$$

Let $\left(\alpha_{1}, \alpha_{2}\right) \in \mathbb{Z}^{2}$ such that $n_{1} \alpha_{1}+n_{2} \alpha_{2} \equiv-w \bmod n_{3}$. Thus, $\mathbb{Z} \backslash \Pi(\mathbf{n}, w)+$ $\left(\alpha_{1}, \alpha_{2}\right)=\boldsymbol{\Pi}\left(\mathbf{n}, n_{3}-w\right)$ and $\boldsymbol{\Pi}(\mathbf{n}, w)$ is 0-connected if and only if $\boldsymbol{\Pi}\left(\mathbf{n}, n_{3}-w\right)$ is not 0 -separating. Since $\boldsymbol{\Pi}\left(\mathbf{n}, w_{0}\right)$ (resp. $\left.\boldsymbol{\Pi}\left(\mathbf{n}, w_{0}-1\right)\right)$ is 0 -connected (resp. is not 0 -connected), then $\boldsymbol{\Pi}\left(\mathbf{n}, n_{3}-w_{0}\right)$ (resp. $\left.\Pi\left(\mathbf{n}, n_{3}-w_{0}+1\right)\right)$ is not 0-separating (resp. is 0 -separating). Hence $\bar{w}_{0}=n_{3}-w_{0}+1$ and the result follows.

Since the $\kappa$-connectedness and the $\kappa$-separatingness of a rational arithmetic discrete plane do not depend on the translation parameter, an easy computation gives the equivalent reformulation of Theorem 6 .

Theorem 7 (Arithmetic reduction). Let $\mathbf{n} \in \mathbb{Z}^{3}$ such that $0 \leq 2 n_{1} \leq 2 n_{2} \leq$ $n_{3}$ and let $w \in \mathbb{Z}$. Let $(q, r) \in \mathbb{N}^{2}$ be the unique pair of integers such that $n_{2}=q n_{1}+r$ and $r \in\left[0, n_{1}\left[\right.\right.$. Let $\mathbf{n}^{\prime}=M \cdot \mathbf{n}$ with

$$
M=\left(\begin{array}{rrr}
1 & 0 & 0 \\
-q & 1 & 0 \\
1-q & -1 & 1
\end{array}\right)
$$

and let $w^{\prime}=w-q n_{1}$. Then, $\boldsymbol{\Pi}(\mathbf{n}, w)$ is 0-separating if and only if so is $\mathbf{\Pi}\left(\mathbf{n}^{\prime}, w^{\prime}\right)$.

Proof (sketch). For clarity, let us first introduce a quite natural notation. One naturally represents a 1 -path $\gamma$ in $\Pi(\mathbf{n}, w)$ as a triple $(A, u, B)$ with:

i) $A \in[0, w[$ (resp. $B \in[0, w[$ ) is the starting (resp. the ending) remainder of the 1-path $\gamma$.

ii) $u \in\left\{ \pm n_{1}, \pm n_{2}, \pm\left(n_{1}-n_{3}\right), \pm\left(n_{2}-n_{3}\right)\right\}^{k}$ is a finite sequence of movements 


\begin{tabular}{|c|c|c|c|c|c|c|c|c|}
\hline 4 & 8 & 12 & 0 & 4 & 8 & 12 & 0 & 4 \\
\hline 13 & 1 & 5 & 9 & 13 & 1 & 5 & 9 & 13 \\
\hline 6 & 10 & 14 & 2 & 6 & 10 & 14 & 2 & 6 \\
\hline 15 & 3 & 7 & 11 & 15 & 3 & 7 & 11 & 15 \\
\hline 8 & 12 & 0 & 4 & 8 & 12 & 0 & 4 & 8 \\
\hline 1 & 5 & 9 & 13 & 1 & 5 & 9 & 13 & 1 \\
\hline
\end{tabular}

Fig. 4. A 1-path corresponding to the triple $\left(1,\left[-n_{2}, n_{1}, n_{1}-n_{3},-\left(n_{2}-\right.\right.\right.$ $\left.\left.\left.n_{3}\right),-n_{2}, n_{1}, n_{1}, n_{1}-n_{3}, n_{1}\right], 4\right)$

between $A$ and $B$ (see Figure 4) (the integer $k \in \mathbb{N}$ is called the length of $u$ ). Let us notice that the movements $\pm\left(n_{1}-n_{3}\right)$ and $\pm\left(n_{2}-n_{3}\right)$ corresponds to horizontal (resp. vertical) movements in $\Pi(\mathbf{n}, w)$ with a change of height in $\mathbf{P}(\mathbf{n}, w)$. Such a change is represented by a thick line in the array of remainders (see Figure 4).

Conversely, let $(A, u, B)$ be a triple with $(A, B) \in\left[0, w\left[^{2}\right.\right.$ and $u \in$ $\left\{ \pm n_{1}, \pm n_{2}, \pm\left(n_{1}-n_{3}\right), \pm\left(n_{2}-n_{3}\right)\right\}^{k}$, with $k \in \mathbb{N}$, then $(A, u, B)$ is a 1-path in $\Pi(\mathbf{n}, w)$ if and only if, for all $j \in\{0, \ldots, k\}, A+\sum_{i=1}^{j} u_{k} \in[0, w[$.

The aim of this proof is to show that $\Pi(\mathbf{n}, w)$ admits an infinite 1-path if and only if so does $\Pi\left(\mathbf{n}^{\prime}, w^{\prime}\right)$.

Let us first prove that each pair of two 1-adjacent pixels in $\Pi\left(\mathbf{n}^{\prime}, w^{\prime}\right)$ can be expanded into a 1-path in $\Pi(\mathbf{n}, w)$.

i) Let $\left(A, n_{1}^{\prime}, B\right)$ represent a pair of two 1-adjacent pixels in $\Pi\left(\mathbf{n}^{\prime}, w^{\prime}\right)$. Then $0 \leq A<w^{\prime}=w-q n_{1} \leq w, 0 \leq B<w^{\prime}=w-q n_{1} \leq w$ and $\left(A, n_{1}, B\right)=$ $\left(A, n_{1}^{\prime}, B\right)$ is a 1 -path in $\Pi(\mathbf{n}, w)$.

ii) Let $\left(A, n_{1}^{\prime}-n_{3}^{\prime}, B\right)$ represent a pair of two 1 -adjacent pixels in $\Pi\left(\mathbf{n}^{\prime}, w^{\prime}\right)$. Since $n_{1}^{\prime}-n_{3}^{\prime}=q n_{1}+n_{2}-n_{3}$ and $A<w-q n_{1}$, then $0 \leq A+q n_{1}<w$ and $(A, \underbrace{n_{1}, \ldots, n_{1}}_{q}, n_{2}-n_{3}, B)$ is a 1 -path in $\operatorname{Pi}(\mathbf{n}, w)$.

The other cases, namely $\left(A, n_{2}^{\prime}, B\right)$ and $\left(A, n_{2}^{\prime}-n_{3}^{\prime}, B\right)$, are obtained in the same way. For summarize, see Figure 5 for a correspondance between a 1-path in $\Pi\left(\mathbf{n}^{\prime}, w^{\prime}\right)$ and a 1-path in $\Pi(\mathbf{n}, w)$. Conversely, if $\Pi(\mathbf{n}, w)$ admits an infinite 1path, then by a similar recoding of it, one obtains an infinite 1-path in $\Pi\left(\mathbf{n}^{\prime}, w^{\prime}\right)$. The complete proof if this theorem will appear in a forthcoming long version of the present paper.

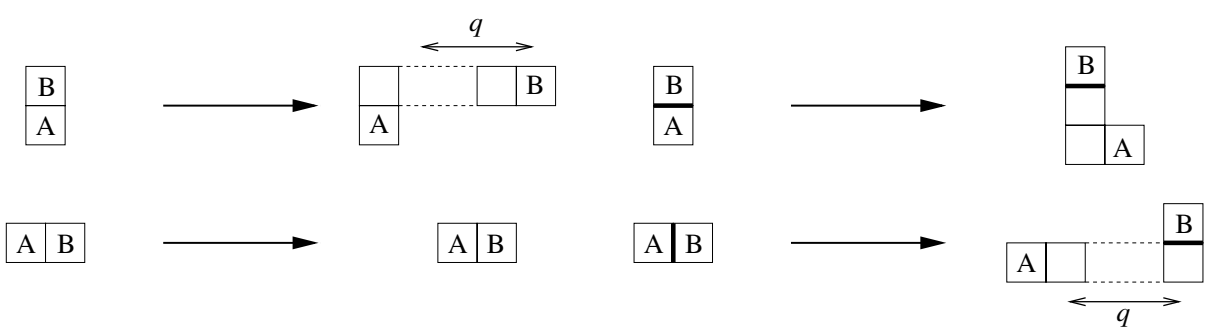

Fig. 5. Transformation of 1-paths in $\Pi\left(\mathbf{n}^{\prime}, w^{\prime}\right)$ into 1-paths in $\Pi(\mathbf{n}, w)$ 


\section{Algorithm}

In the present section, we design an algorithm which computes the 0 -connecting thickness of a given integer vector $\mathbf{n} \in \mathbb{Z}^{3}$. It iterates the reduction introduced in Theorem [ 6 until 0-connecting thickness becomes easy to determine.

The arithmetic reduction mentioned above only preserves 0 -connectedness between the arithmetic discrete plane with normal vector $\mathbf{n}$ and its image under some conditions on $\mathbf{n}$. Nevertheless changing the components of a vector according to the symmetry lemma 3 or sorting them do not change the associated 0 -connecting thickness. It is then possible to find from any vector $\mathbf{n}$ a vector $\mathbf{n}^{\prime}$ with the same 0-connecting thickness meeting the requirement of Theorem 6. A step consisting of application of symmetry lemma, sorting, and the arithmetic reduction can be repeated and turns the vector $\mathbf{n}$ into another vector $\mathbf{n}^{\prime}$ such that $n_{1} \leq n_{1}^{\prime}, n_{2}<n_{2}^{\prime}$ and $n_{3}<n_{3}^{\prime}$. Consequently, after a finite number of iteration, we always obtain a vector with a zero component for which the 0 -connecting thickness is easy to determine.

Algorithm 1. follows from those considerations. It always terminates since the stopping condition, that is a vector with a zero component, is always reached in a finite number of iteration.

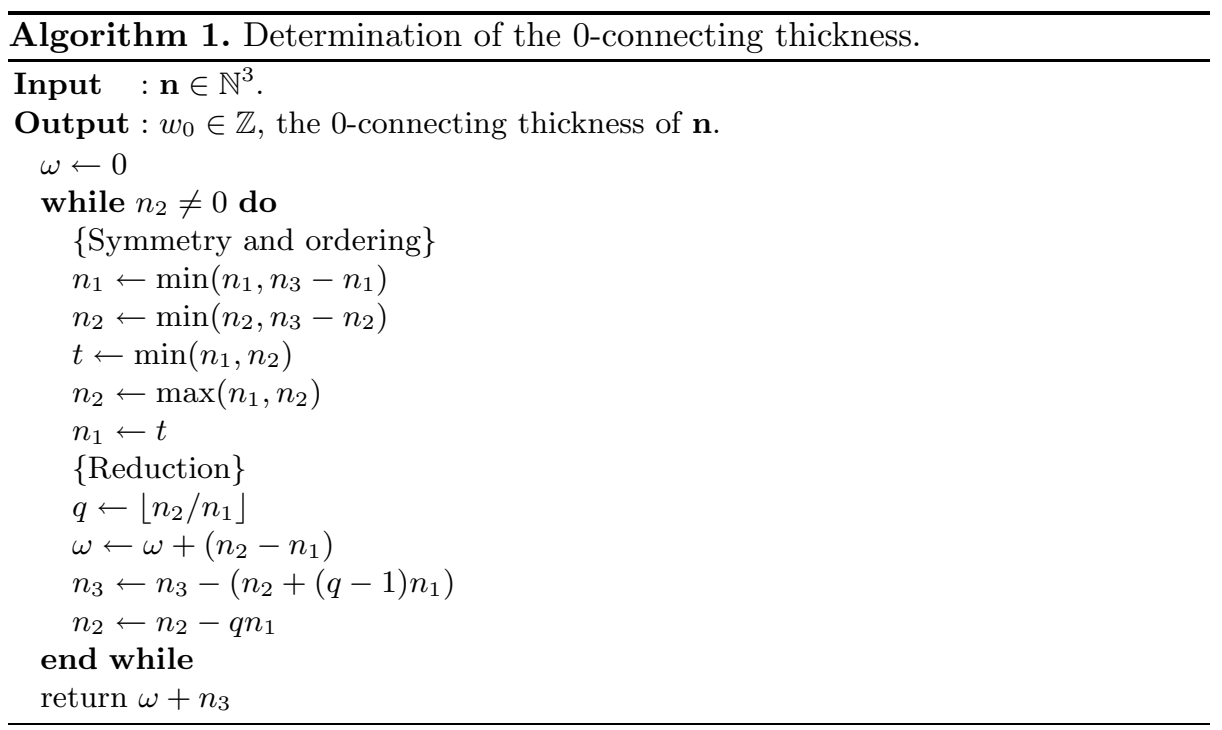

\section{Conclusion and Perspectives}

In the present paper, we presented an algorithm computing the 0-connecting thickness of any integer vector. The main difference between this algorithm and the ones already known [43] is that it does not need a graph traversal and only computes basic reductions on an integer vector. 
In a forthcoming work, we plan to investigate the case of non-rational arithmetic planes. Since the reduction of Theorem 6 does not depend on the nature of the input vector (integer or not), we hope to extend this approach to any vector $\mathbf{n} \in \mathbb{R}^{3}$.

Other interesting investigations should be, on the one hand, the computation of $\kappa$-connected thickness for $\kappa \in\{1,2\}$ and, on the other, the extension of this work to arithmetic discrete hyperplanes in any dimension.

\section{Acknowledgements}

We would like to thank E. Andres for having pointed out the original problem to the authors. We also thank P. Arnoux, V. Berthe, V. Brimkov, C. Fiorio, Y. Gérard and D. Vergnaud for many interesting discussions on the subject.

\section{References}

1. Reveillès, J.P.: Géométrie discrète, Calcul en Nombres Entiers et Algorithmique. Thèse d'Etat, Université Louis Pasteur, Strasbourg (1991)

2. Andres, E., Acharya, R., Sibata, C.: Discrete analytical hyperplanes. CVGIP: Graphical Models and Image Processing 59(5) (1997) 302-309

3. Gérard, Y.: Periodic graphs and connectivity of the rational digital hyperplanes. Theoritical Computer Science 283(1) (2002) 171-182

4. Brimkov, V., Barneva, R.: Connectivity of discrete planes. Theoritical Computer Science 319(1-3) (2004) 203-227

5. Debled-Rennesson, I.: Etude et reconnaissance des droites et plans discrets. Thèse de Doctorat, Université Louis Pasteur, Strasbourg. (1995) 